\title{
Asexual and sexual morphs of Moesziomyces revisited
}

\author{
Julia Kruse ${ }^{1,2}$, Gunther Doehlemann ${ }^{3}$, Eric Kemen, and Marco Thines ${ }^{1,2,5}$
}

${ }^{1}$ Goethe University, Department of Biological Sciences, Institute of Ecology, Evolution and Diversity, Max-von-Laue-Str. 13, D-60486 Frankfurt am Main, Germany; corresponding author e-mail: thines@smut-fungi.net

${ }^{2}$ Biodiversität und Klima Forschungszentrum, Senckenberg Gesellschaft für Naturforschung, Senckenberganlage 25, D-60325 Frankfurt am Main, Germany

${ }^{3}$ Botanical Institute and Center of Excellence on Plant Sciences (CEPLAS), University of Cologne, BioCenter, Zülpicher Str. 47a, D-50674, Köln, Germany

${ }^{4}$ Max Planck Institute for Plant Breeding Research, Carl-von-Linne-Weg 10, 50829 Köln, Germany

${ }^{5}$ Integrative Fungal Research Cluster (IPF), Georg-Voigt-Str. 14-16, D-60325 Frankfurt am Main, Germany

Abstract: Yeasts of the now unused asexually typified genus Pseudozyma belong to the smut fungi (Ustilaginales) and are mostly believed to be apathogenic asexual yeasts derived from smut fungi that have lost pathogenicity on plants. However, phylogenetic studies have shown that most Pseudozyma species are phylogenetically close to smut fungi parasitic to plants, suggesting that some of the species might represent adventitious isolations of the yeast morph of otherwise plant pathogenic smut fungi. However, there are some species, such as Moesziomyces aphidis (syn. Pseudozyma aphidis) that are isolated throughout the world and sometimes are also found in clinical samples and do not have a known plant pathogenic sexual morph. In this study, it is revealed by phylogenetic investigations that isolates of the biocontrol agent Moesziomyces aphidis are interspersed with $M$. bullatus sexual lineages, suggesting conspecificity. This raises doubts regarding the apathogenic nature of asexual morphs previously placed in Pseudozyma, but suggests that there might also be pathogenic sexual morph counterparts for those species known only from asexual morphs. The finding that several additional species currently only known from their yeast morphs are embedded within the genus Moesziomyces, suggests that the yeast morph might play a more dominant role in this genus as compared to other genera of Ustilaginaceae. In addition, phylogenetic reconstructions demonstrated that Moesziomyces bullatus has a narrow host range and that some previously described but not widely used species names should be applied for Moesziomyces on other host genera than Echinochloa.
Key words: ecology evolution phylogeny plant pathogens pleomorphic fungi Ustilaginomycotina yeast

Article info: Submitted: 7 December 2017; Accepted: 4 May 2017; Published: 15 May 2017.

\section{INTRODUCTION}

Ustilaginales is the largest order within the smut fungi (Ustilaginomycetes), including species forming a blackish to brownish powdery spore mass in different organs of monocotyledonous, and exceptionally dicotyledonous plants (Vánky 2012, Begerow et al. 2006). The order includes nine families, encompassing 54 genera. The Anthracoideaceae with the occurrence on Cyperaceae and Juncaceae, and Ustilaginaceae, with few exceptions parasitic to the Poaceae, are the largest families within the order. The latter contains the three largest smut genera, Anthracocystis, Sporisorium, and Ustilago. The difference between these three closely related genera is the almost complete lack of a plant-derived columella within sori formed by Ustilago species (Vánky 2012, McTaggart et al. 2012). Within Ustilago some species are economically important pathogens, like corn smut (Ustilago maydis) or wheat smut (Ustilago nuda). Ustilago maydis is a species for which one of the first fungal genomes was sequenced (Kämper et al. 2006). Smut fungi of the
Ustilaginales usually feature both an asexual yeast morph and a sexual morph infecting host plants in a biotrophic manner. In rare cases yeasts of the Ustilaginales could also be found to be affecting humans (McNeil \& Palazzi 2012, Teo \& Tay 2006). The earliest case of an invasive infection with an Ustilago species, possibly $U$. maydis, was reported in 1946 (Moore et al. 1946). But spores of the Ustilaginales potentially also cause pneumonias, allergic reactions, or asthma (Valverde et al. 1995).

There are several studies dealing with the phylogeny of Ustilaginomycotina, mostly based on the LSU or ITS locus and some of them include asexual morphs as well (e.g. Begerow et al. 2000, 2006, Stoll et al. 2005, Wang et al. 2006, 2015, Boekhout 2011). Wang et al. (2015) link many asexual yeasts to their corresponding sexual morphs, an important step within the naming of pleomorphic fungi, as dual naming of sexual and asexual morphs is now discontinued (Hawksworth et al. 2011).

Pseudozyma has been used for species of ustilaginomycetous yeasts belonging to Ustilaginales which are mostly

\section{2017 International Mycological Association}

You are free to share - to copy, distribute and transmit the work, under the following conditions:

Attribution: $\quad$ You must attribute the work in the manner specified by the author or licensor (but not in any way that suggests that they endorse you or your use of the work).

Non-commercial: You may not use this work for commercial purposes.

No derivative works: You may not alter, transform, or build upon this work.

For any reuse or distribution, you must make clear to others the license terms of this work, which can be found at http://creativecommons.org/licenses/by-nc-nd/3.0/legalcode. Any of the above conditions can be waived if you get permission from the copyright holder. Nothing in this license impairs or restricts the author's moral rights. 
believed to be apathogenic (Begerow et al. 2000, 2006). The genus was described in 1985, by Bandoni (1985), and later refined by Boekhout (1995). After Sampaio (2004) and finally Wang et al. (2015) established that the type species Pseudozyma prolifica was a synonym of Ustilago maydis, the name Pseudozyma should no longer be used. However, the phylogenetic position of some species referred to Pseudozyma is still unclear. Wang et al. (2015) suggested using the name Pseudozyma now with the addition "pro tempore" for five Pseudozyma species with an unclear phylogenetic position. In the current study we give Pseudozyma names with reference to the new combinations recently made, where possible.

To date, about 20 Pseudozyma species names were validly published (www.indexfungroum.org). Fifteen are now linked to a corresponding sexually typified genus (Wang et al. 2015). Of these, Pseudozyma antarctica, $P$. aphidis, $P$. parantarctica, and $P$. rugulosa were transferred to Moesziomyces (Wang et al. 2015).

Ustilaginalean yeasts are isolated from diverse habitats, for Ustilaginales, but mostly from grasses (Boekhout 1995, Avis et al. 2001). Some were isolated from flowers, leaves or fruits of other plants, but it is also possible to isolate them from soil or even human blood or secretory fluid (Sugita et al. 2003, Arendrup et al. 2014). Apart from the few clinical cases of Ustilago maydis infestation, it was not known until 2003 that species referred to Pseudozyma could also infect humans (Sugita et al. 2003). However, such species can cause invasive infections, especially in immunosuppressed individuals (Arendrup et al. 2014). The infection risk, according to Prakash et al. (2014), is the same as being colonised by nonalbicans Candida infections. Furthermore, Avis et al. (2001) and Gafni et al. (2015) reported antifungal properties of some pseudozyma-like yeast species, including Moesziomyces aphidis, and some strains have been reported to be natural antagonists of powdery mildews (Erysiphales). Colonization of leaf surfaces by these yeasts provides a natural source of protection against some plant pathogenic fungi (Gafni et al. 2015).

Of the ustilaginalean yeasts, especially Moesziomyces antarcticus and $M$. aphidis, formerly treated as Pseudozyma species, have been frequently isolated from various substrates. Moesziomyces antarcticus was isolated from plants and soil, but also from blood of humans (Boekhout 2011). Moesziomyces aphidis was described in 1995 and first isolated from the secretion of an aphid, but it has later been isolated from water (Boekhout 2011) and various other sources, including soil and human blood. Wang et al. (2015) showed that these pseudozyma-like species, together with $M$. parantarcticus and $M$. rugulosus, belong to the genus Moesziomyces, which before had been generally regarded as monotypic (Vánky 2005).

Moesziomyces mainly differs from other smut fungi in having remnants of ruptured sterile cells (Vánky 1977). Vánky (1977) initially included four species in the genus Moesziomyces. Three of them, occurring on different genera of grasses (Leersia, Paspalum, Pennisetum) were later considered to be conspecific and united under the oldest name, M. bullatus (Vánky 1986, 2005). The other species, M. eriocauli (Vánky 1986), was transferred to a new genus
Eriomoeszia, because of a thin cortex of sterile cells, which surrounds the spore balls (Vánky 2005). After the transfer of four pseudozyma-like species ( $M$. antarcticus, $M$. aphidis, $M$. parantarcticus and $M$. rugulosus) to this genus, it now contains five species (Wang et al. 2015).

Given the high host specificity observed for most Ustilaginales species (McTaggart et al. 2012, Escudero 2015, Li et al. 2017), it seemed doubtful that Moesziomyces bullatus was parasitic to seven not closely related genera, suggesting that more species might be present in the genus, some of which might be conspecific with smuts in the past named in Pseudozyma. It was the aim of the current study to clarify the relationships of asexual and sexual morphs in the genus Moesziomyces.

\section{MATERIAL AND METHODS}

\section{Fungal material}

The fungal material used in this study is listed in Tables 1 and 2. The nomenclature of the hosts is derived from the latest version of The International Plant Names Index (www.ipni. org), the nomenclature of the fungi follows Vánky (2012) and MycoBank (www.mycobank.org).

\section{Yeast cultivation}

Fresh material of Moesziomyces bullatus collected in 2015 (GLM-F105817) was used for yeast cultivation. A suspension of spores in $2 \mathrm{~mL}$ water was prepared. Three tubes with $200 \mu \mathrm{L}$ spore suspension were exposed to three different conditions: (1) heating of the suspension on a thermomixer at $45{ }^{\circ} \mathrm{C}$ for $5 \mathrm{~min}$ (Shetty \& Safeeulla 1979); (2) chilling the suspension overnight on ice; and (3) incubation for $5 \mathrm{~min}$ at room temperature (ca. $20{ }^{\circ} \mathrm{C}$ ). From each tube $20 \mu \mathrm{L}$ suspension was each plated on two plates of SAM (Thines lab Standard Agar Medium, consisting of $20 \mathrm{~g}$ agar, $20 \mathrm{~g}$ PDB, 10 $\mathrm{g}$ yeast extract, $10 \mathrm{~g}$ malt extract, $40 \mathrm{~mL}$ clarified vegetable juice, $960 \mathrm{~mL}$ water) with the addition of $75 \mathrm{mg}$ Rifampicin/L. One set of plates was incubated at $30{ }^{\circ} \mathrm{C}$, the other set at room temperature. After $3 \mathrm{~d}$ on every plate abundant yeast growth was recognized. Pure cultures were produced by picking and transferring individual single colonies to the SAM medium (isolate $\mathrm{A} 1-\mathrm{A} 10$ ). To isolate pseudozyma-morphs associated with Albugo laibachii on Arabidopsis thaliana, A. laibachii spores were suspended in water and treated with antibiotics to remove bacteria. Subsequently, the suspension was plated on PDA at $20^{\circ} \mathrm{C}$ and colonies were singled out after $7 \mathrm{~d}$.

\section{DNA extraction, PCR and sequencing}

In total 5-20 mg of infected plant tissue from herbarium specimens and yeast colonies were disrupted in a mixer mill (MM2, Retsch), using two iron beads of $3 \mathrm{~mm}$ and 5-8 iron beads of $1 \mathrm{~mm}$ diam per sample and shaking at $25 \mathrm{~Hz}$ for 5-10 min. Genomic DNA was extracted using the BioSprint 96 DNA Plant Kit (Qiagen, Hilden) on a KingFisher Flex robot (Thermo Scientific, Dreieich). PCR amplification of the complete ITS nrDNA was performed using the M-ITS1 forward primer (Stoll et al. 2003) and the ITS4 (White et al. 1990) or smITS-R1 reverse primer (Kruse et al. 2017). 
Table 1. List of Moesziomyces specimens used in the present study.

\begin{tabular}{|c|c|c|c|c|c|c|c|}
\hline Species & Host & Host family & Location & Year & Collector & Fungarium no. & ITS GenBank acc. no. \\
\hline Moesziomyces aphidis & $\begin{array}{l}\text { Arabidopsis thaliana infected with } \\
\text { Albugo laibachii }\end{array}$ & Brassicaceae & UK, Norwich & 2007 & E. Kemen & "GLM-F107578 & KY930224 \\
\hline \multirow[t]{16}{*}{ Moesziomyces bullatus } & Echinochloa crus-galli & Poaceae & Germany, Bavaria & 2013 & J. Kruse & GLM-F105812 & KY424439 \\
\hline & Echinochloa crus-galli & Poaceae & Germany, Bavaria & 2013 & J. Kruse & GLM-F105813 & KY424428 \\
\hline & Echinochloa crus-galli & Poaceae & Germany, Bavaria & 2013 & J. Kruse & GLM-F105814 & KY424429 \\
\hline & Echinochloa muricata & Poaceae & Germany, Saxony & 2000 & H. Jage & GLM-F047045 & KY424430 \\
\hline & Echinochloa muricata & Poaceae & Germany, Saxony & 2000 & $\begin{array}{l}\text { D. Schulz, B. Huber } \\
\text { \& F. Klenke }\end{array}$ & GLM-F047047 & KY424431 \\
\hline & Echinochloa muricata & Poaceae & Germany, Saxony-Anhalt & 2003 & H. Jage & GLM-F065276 & KY424432 \\
\hline & Echinochloa muricata & Poaceae & Germany, Saxony-Anhalt & 2005 & H. Jage & GLM-F076000 & KY424433 \\
\hline & Echinochloa crus-galli & Poaceae & Germany, North Rhine-Westphalia & 2010 & J. Kruse & GLM-F105815 & KY424434 \\
\hline & Echinochloa crus-galli & Poaceae & Germany, Schleswig-Holstein & 2011 & J. Kruse & GLM-F105777 & KY424435 \\
\hline & Echinochloa crus-galli & Poaceae & Poland & 1979 & K. Vánky & $\begin{array}{l}\text { HUV No. } 283 \text {, ex } \\
\text { TUB }\end{array}$ & KY424436 \\
\hline & Echinochloa crus-galli & Poaceae & Germany, Hesse & 2015 & J. Kruse & ex-GLM-F105817 & KY424437 \\
\hline & & & & & & $\begin{array}{l}\text { culture No. A1 } \\
\text { GLM-F107575 }\end{array}$ & \\
\hline & Echinochloa crus-galli & Poaceae & Germany, Hesse & 2015 & J. Kruse & ex-GLM-F105817 & KY424427 \\
\hline & & & & & & $\begin{array}{l}\text { culture No. A3 } \\
\text { GLM-F107576 }\end{array}$ & \\
\hline & Echinochloa crus-galli & Poaceae & Germany, Hesse & 2015 & J. Kruse & ex-GLM-F105817 & KY424438 \\
\hline & & & & & & $\begin{array}{l}\text { culture No. A10 } \\
\text { GLM-F107577 }\end{array}$ & \\
\hline Moesziomyces penicillariae & Pennisetum glaucum & Poaceae & Westafrica, Gambia & 1973 & K. Vánky & $\begin{array}{l}\text { HUV No. } 154 \text {, ex } \\
\text { TUB }\end{array}$ & KY424440 \\
\hline
\end{tabular}


Table 2. List of additional sequences used in the phylogenetic tree, downloaded from GenBank.

\begin{tabular}{|c|c|c|c|}
\hline Species & Source & ITS GenBank acc. no. & Citation \\
\hline Eriomoeszia eriocauli & Eriocaulon cinereum & AY740041 & Stoll et al. (2005) \\
\hline Macalpinomyces eriachnes & Eriachne helmsii & AY740038 & Stoll et al. (2005) \\
\hline \multirow[t]{5}{*}{ Moesziomyces bullatus } & Paspalum distichum & AY74015)3 & Stoll et al. (2005) \\
\hline & human preterm low birth weight infant & KF926673 & Okolo et al. (2015) \\
\hline & - & DQ831013 & Matheny et al. (2006) \\
\hline & human preterm low birth weight infant & KF926673 & Okolo et al. (2015) \\
\hline & - & DQ831013 & Matheny et al. (2006) \\
\hline \multirow[t]{5}{*}{ Pseudozyma antarctica } & - & JX094775 & Gujjari et al. (unpublished) \\
\hline & - & JN942669 & An (unpublished) \\
\hline & unpolished Japanese rice & AB089360 & Sugita et al. (2003) \\
\hline & Antarctica sediment & AF294698 & Avis et al. (2001) \\
\hline & lake sediment & AB089358 & Sugita et al. (2003) \\
\hline \multirow[t]{22}{*}{ Pseudozyma aphidis } & Japanese pear fruit & AB204896 & Yasuda et al. (2007) \\
\hline & human pulmonary infection & Q743064 & Parahym et al. (2013) \\
\hline & Saccharum officinarum & AB704889 & Morita et al. (2012) \\
\hline & Leucaena glauca & HQ662536 & Wei et al. (2011) \\
\hline & human & EU105)207 & Lin et al. (2008) \\
\hline & human blood & AB089362 & Sugita et al. (2003) \\
\hline & human & HQ848933 & Xie et al. (unpublished) \\
\hline & Fallopia japonica & KC282385 & Wang \& Liu (unpublished) \\
\hline & blood culture from hospitalized patient & KM610219 & $\begin{array}{l}\text { Bosco-Borgeat \& Taverna } \\
\text { (unpublished) }\end{array}$ \\
\hline & poplar leaf & KM268868 & Sun \& Yan (unpublished) \\
\hline & Forcipomia taiwana & KM555221 & Chen (unpublished) \\
\hline & seaweeds & KP269028 & Wang et al. (unpublished) \\
\hline & aphid secretion & AF294699 & Avis et al. (2001) \\
\hline & Neoreglia cruenta & FN424100 & Garcia et al. (unpublished) \\
\hline & Saccharum officinarum & AB704878 & Morita et al. (2012) \\
\hline & giant panda secrete & KF973199 & Li et al. (unpublished) \\
\hline & Camellia sinensis foliar lesions & HQ832804 & Li et al. (unpublished) \\
\hline & Echinochloa crus-galli & GU390690 & Hamayun \& Ahmad (unpublished) \\
\hline & aphid secretion on Solanum pseudocapsicum & JN942666 & An (unpublished) \\
\hline & mulberry leaf & KF443199 & Qiu et al. (unpublished) \\
\hline & Citrus leaf & JQ425372 & Soliman (unpublished) \\
\hline & - & JN942667 & An (unpublished) \\
\hline Pseudozyma hubeiensis & Magnolia denudata wilting leaf & DQ008954 & Wang et al. (2006) \\
\hline \multirow[t]{5}{*}{ Pseudozyma parantarctica } & - & JN544036 & Chen (unpublished) \\
\hline & yam tuber steep water & KF619567 & Babajide et al. (2015) \\
\hline & - & KP132543 & Irinyi et al. (2015) \\
\hline & human blood & AB089356 & Sugita et al. (2003) \\
\hline & - & NR 130693 & An (unpublished) \\
\hline \multirow[t]{3}{*}{ Pseudozyma rugulosa } & mouldy Zea mays leaf & AB089370 & Sugita et al. (2003) \\
\hline & ex-leaf of corn & AF294697 & Avis et al. (2001) \\
\hline & plant leaf & HE650886 & Han et al. (2002) \\
\hline
\end{tabular}


Table 2. (Continued)

\begin{tabular}{llll}
\hline Species & Source & ITS GenBank acc. no. & Citation \\
\hline \hline & Coffea arabica & EU002890 & Vega et al. (unpublished) \\
& Hyoscyamus muticus & AB500690 & Abdel-Motaal \& Itu (unpublished) \\
& Soffea arabica & DQ778919 & Vega et al. (2008) \\
& shoot of tip pepper & LC05)3989 & Surussawadee \& Limtong \\
(unpublished)
\end{tabular}

* type collections are highlighted in bold

The reaction was performed in a thermocycler (Eppendorf Mastercycler 96 vapo protect, Eppendorf, Hamburg) with an initial denaturation at $95{ }^{\circ} \mathrm{C}$ for $4 \mathrm{~min}, 36 \mathrm{PCR}$ cycles of denaturation at $95{ }^{\circ} \mathrm{C}$ for $40 \mathrm{~s}$, annealing at $56{ }^{\circ} \mathrm{C}$ for $40 \mathrm{~s}$ and elongation at $72^{\circ} \mathrm{C}$ for $60 \mathrm{~s}$, followed by a final elongation at $72^{\circ} \mathrm{C}$ for $4 \mathrm{~min}$. The resulting amplicons were sequenced at the Biodiversity and Climate Research Centre (BiK-F) laboratory using the PCR primers. Sequences were deposited in GenBank (NCBI, Table 1).

\section{Phylogenetic reconstructions}

The dataset included sequences of Moesziomyces species sexual and asexual morphs, both newly sequenced (Table 1) and downloaded from GenBank (Table 2). First all available sequences were extracted from $\mathrm{NCBI}$ on the basis of sequence similarity. Subsequently sequences were removed that were: (1) highly redundant with already-included ITS genotypes; or (2) of doubtful sequence quality, i.e. with mutations at positions highly conserved or with nucleotide changes only towards one end of the sequences.

Macalpinomyces eriachnes was selected as an outgroup, based on the phylogenetic tree in Shivas et al. (2013). Alignments were made using mafft v. 7 (Katoh \& Standley 2013) employing the Q-INS-I algorithm and removing leading and trailing gaps. The resulting total alignment length was 576 bp. For phylogenetic analyses, Minimum Evolution (ME) analysis was done with Mega v. 6.06 (Tamura et al. 2013), using the Tamura-Nei substitution model, assuming partial deletion at a cut-off of $80 \%$ and 1000 bootstrap replicates. Maximum Likelihood (ML) analysis was done using RAxML on the webserver TrEase (www.thines-lab.senckenberg.de/ trease) with all parameters were set to default values. For Bayesian analysis also the webserver TrEase was used for calculating $10 \mathrm{M}$ tree generations on four incrementally heated MC chains. The first $30 \%$ of the trees obtained this way were discarded to ensure sampling of the stationary phase. All other parameters were set to default.

\section{Morphological examination}

For light microscopy, the herbarium specimens GLM-F105814 and GLM-F105812 were transferred to distilled water on a slide. Morphological examination was carried out using a Zeiss Imager M2 AX10 microscope (Carl Zeiss, Göttingen). Measurements of the spore balls and spores were performed at $\times 400$. Measurements are reported as maxima and minima in parentheses, and the mean plus and minus the standard deviation of a number of measurements given in parenthesis; the means are given in italics (Table 3 ).

\section{RESULTS}

The isolated yeasts from fresh Moesziomyces bullatus samples from Echinochloa crus-galli and E. muricatus were fast-growing on SAM medium. The colour of the yeasts was cream to light reddish, and the shape of the colonies was regular and roundish.

A phylogenetic hypothesis for the sampled Moesziomyces species and the cultivated yeast asexual morphs is given in Fig. 1. The results of the Minimum Evolution, Maximum Likelihood and Bayesian Analyses were congruent. The clade comprising the type of $M$. aphidis also includes $M$. bullatus s. str. from Echinochloa crus-galli and E. muricata, as well as the sequence of the type of $M$. rugulosa, an isolate of Moesziomyces from Albugo laibachii on Arabidopsis thaliana and many other isolates not determined to the species level from various sources. While visual inspection of the alignments revealed that there was some sequence variation within the Moesziomyces bullatus clade, the relationships of the four subgroups was not resolved apart from the clustering 
Table 3. Measurements from 25 spore balls and 100 teliospores for collections of Moesziomyces bullatus on Echinochloa crus-galli from two different clades.

\begin{tabular}{|c|c|c|c|c|c|c|c|c|c|c|c|c|}
\hline \multirow[b]{3}{*}{ No. } & \multicolumn{6}{|c|}{$\begin{array}{l}\text { Moesziomyces bullatus ex Echinochloa crus-galli, } \\
\text { GLM-F105814 }\end{array}$} & \multicolumn{6}{|c|}{$\begin{array}{l}\text { Moesziomyces bullatus ex Echinochloa crus-galli, } \\
\text { GLM-F105812 }\end{array}$} \\
\hline & \multicolumn{3}{|c|}{ sporeballs } & \multicolumn{3}{|c|}{ spores } & \multicolumn{3}{|c|}{ sporeballs } & \multicolumn{3}{|c|}{ spores } \\
\hline & length & breadth & $1 / \mathrm{b}$ & length & breadth & $\mathrm{l} / \mathrm{b}$ & length & breadth & $1 / b$ & length & breadth & $\mathrm{l} / \mathrm{b}$ \\
\hline 1 & 148,5 & 100 & 1,49 & 7,5 & 7,5 & 1 & 120,5 & 76,5 & 1,58 & 8,5 & 7 & 1,21 \\
\hline 2 & 111 & 91 & 1,22 & 8 & 7,5 & 1,07 & 81 & 68,5 & 1,18 & 8 & 7,5 & 1,07 \\
\hline 3 & 79 & 65 & 1,22 & 7 & 7 & 1 & 64,5 & 55,5 & 1,16 & 7,5 & 7 & 1,07 \\
\hline 4 & 118,5 & 97,5 & 1,22 & 8,5 & 7 & 1,21 & 58,5 & 53 & 1,1 & 8 & 7 & 1,14 \\
\hline 5 & 58,5 & 58,5 & 1 & 8 & 6 & 1,33 & 97 & 53,5 & 1,81 & 7,5 & 7,5 & 1 \\
\hline 6 & 100,5 & 83,5 & 1,2 & 8 & 7,5 & 1,07 & 76 & 61,5 & 1,24 & 8 & 6 & 1,33 \\
\hline 7 & 125 & 113 & 1,11 & 7,5 & 7 & 1,07 & 94 & 87 & 1,08 & 7,5 & 6,5 & 1,15 \\
\hline 8 & 93 & 89,5 & 1,04 & 7,5 & 7 & 1,07 & 61 & 44 & 1,39 & 7,5 & 6,5 & 1,15 \\
\hline 9 & 95,5 & 59 & 1,62 & 8 & 6 & 1,33 & 101,5 & 69 & 1,47 & 7,5 & 6,5 & 1,15 \\
\hline 10 & 82 & 61 & 1,34 & 7 & 7 & 1 & 101 & 74 & 1,36 & 8,5 & 6 & 1,42 \\
\hline 11 & 102 & 69,5 & 1,47 & 9 & 7 & 1,29 & 144,5 & 73,5 & 1,97 & 8 & 6,5 & 1,23 \\
\hline 12 & 53 & 41,5 & 1,28 & 9 & 7 & 1,29 & 84 & 66 & 1,27 & 9 & 7 & 1,29 \\
\hline 13 & 95 & 78 & 1,22 & 8,5 & 6,5 & 1,31 & 90 & 69,5 & 1,29 & 8 & 6,5 & 1,23 \\
\hline 14 & 53 & 41,5 & 1,28 & 7,5 & 6,5 & 1,15 & 83,5 & 61 & 1,37 & 8,5 & 6,5 & 1,31 \\
\hline 15 & 138,5 & 96,5 & 1,44 & 8,5 & 6,5 & 1,31 & 96,5 & 51,5 & 1,87 & 8,5 & 7,5 & 1,13 \\
\hline 16 & 93 & 71,5 & 1,3 & 7 & 7 & 1 & 111,5 & 78,5 & 1,42 & 7,5 & 6,5 & 1,15 \\
\hline 17 & 115 & 91 & 1,26 & 7 & 6,5 & 1,08 & 91,5 & 68,5 & 1,34 & 7 & 6,5 & 1,08 \\
\hline 18 & 71 & 49,5 & 1,43 & 7,5 & 6,5 & 1,15 & 68,5 & 49,5 & 1,38 & 7 & 5,5 & 1,27 \\
\hline 19 & 111,5 & 104 & 1,07 & 7,5 & 7 & 1,07 & 96 & 82,5 & 1,16 & 8,5 & 7 & 1,21 \\
\hline 20 & 117,5 & 95,5 & 1,23 & 7,5 & 7,5 & 1 & 84,5 & 78,5 & 1,08 & 7,5 & 7 & 1,07 \\
\hline 21 & 92,5 & 75,5 & 1,23 & 7,5 & 7 & 1,07 & 86,5 & 62 & 1,4 & 7,5 & 6,5 & 1,15 \\
\hline 22 & 80,5 & 68 & 1,18 & 8 & 7 & 1,14 & 122,5 & 86 & 1,42 & 8,5 & 7,5 & 1,13 \\
\hline 23 & 156,5 & 97,5 & 1,61 & 8 & 7 & 1,14 & 113,5 & 78,5 & 1,45 & 7,5 & 5,5 & 1,36 \\
\hline 24 & 58,5 & 53,5 & 1,09 & 8,5 & 7 & 1,21 & 106 & 105 & 1,01 & 8 & 6 & 1,33 \\
\hline 25 & 52,5 & 52 & 1,01 & 8,5 & 6 & 1,42 & 105,5 & 84,5 & 1,25 & 7 & 6 & 1,17 \\
\hline 26 & & & & 7 & 6,5 & 1,08 & & & & 7 & 7 & 1 \\
\hline 27 & & & & 7,5 & 7,5 & 1 & & & & 6,5 & 6,5 & 1 \\
\hline 28 & & & & 8,5 & 8 & 1,06 & & & & 6,5 & 5,5 & 1,18 \\
\hline 29 & & & & 6,5 & 6 & 1,08 & & & & 8,5 & 7,5 & 1,13 \\
\hline 30 & & & & 7 & 6 & 1,17 & & & & 8 & 6,5 & 1,23 \\
\hline 31 & & & & 7,5 & 6 & 1,25 & & & & 8 & 6 & 1,33 \\
\hline 32 & & & & 8 & 7,5 & 1,07 & & & & 7,5 & 6,5 & 1,15 \\
\hline 33 & & & & 7 & 6,5 & 1,08 & & & & 7 & 6,5 & 1,08 \\
\hline 34 & & & & 7 & 6,5 & 1,08 & & & & 8,5 & 8 & 1,06 \\
\hline 35 & & & & 8,5 & 7,5 & 1,13 & & & & 8 & 7,5 & 1,07 \\
\hline 36 & & & & 7 & 6 & 1,17 & & & & 8 & 7,5 & 1,07 \\
\hline 37 & & & & 7,5 & 6 & 1,25 & & & & 8,5 & 7 & 1,21 \\
\hline 38 & & & & 7 & 6 & 1,17 & & & & 8 & 7 & 1,14 \\
\hline 39 & & & & 7,5 & 6,5 & 1,15 & & & & 8 & 6,5 & 1,23 \\
\hline 40 & & & & 8 & 6,5 & 1,23 & & & & 6,5 & 6 & 1,08 \\
\hline 41 & & & & 6,5 & 6 & 1,08 & & & & 7 & 7 & 1 \\
\hline 42 & & & & 6,5 & 6 & 1,08 & & & & 8,5 & 6 & 1,42 \\
\hline 43 & & & & 8,5 & 6,5 & 1,31 & & & & 7 & 6,5 & 1,08 \\
\hline 44 & & & & 8,5 & 7,5 & 1,13 & & & & 7 & 7 & 1 \\
\hline 45 & & & & 7,5 & 6,5 & 1,15 & & & & 8 & 6,5 & 1,23 \\
\hline 46 & & & & 8,5 & 7 & 1,21 & & & & 8,5 & 7 & 1,21 \\
\hline
\end{tabular}


Table 3. (Continued).

\begin{tabular}{|c|c|c|c|c|c|c|c|c|c|c|c|}
\hline \multirow[b]{3}{*}{ No. } & \multicolumn{5}{|c|}{$\begin{array}{l}\text { Moesziomyces bullatus ex Echinochloa crus-galli, } \\
\text { GLM-F105814 }\end{array}$} & \multicolumn{6}{|c|}{$\begin{array}{l}\text { Moesziomyces bullatus ex Echinochloa crus-galli, } \\
\text { GLM-F105812 }\end{array}$} \\
\hline & \multicolumn{2}{|l|}{ sporeballs } & \multicolumn{3}{|c|}{ spores } & \multicolumn{3}{|c|}{ sporeballs } & \multicolumn{3}{|c|}{ spores } \\
\hline & breadth & $\mathrm{l} / \mathrm{b}$ & length & breadth & $\mathrm{l} / \mathrm{b}$ & length & breadth & $1 / b$ & length & breadth & $\mathrm{l} / \mathrm{b}$ \\
\hline 47 & & & 7 & 7 & 1 & & & & 7,5 & 6,5 & 1,15 \\
\hline 48 & & & 7 & 6,5 & 1,08 & & & & 8 & 7,5 & 1,07 \\
\hline 49 & & & 6,5 & 6,5 & 1 & & & & 8 & 6 & 1,33 \\
\hline 50 & & & 9 & 7 & 1,29 & & & & 7,5 & 7 & 1,07 \\
\hline 51 & & & 8 & 6 & 1,33 & & & & 6 & 5,5 & 1,09 \\
\hline 52 & & & 8 & 6 & 1,33 & & & & 7,5 & 6 & 1,25 \\
\hline 53 & & & 7,5 & 7 & 1,07 & & & & 7 & 7 & 1 \\
\hline 54 & & & 7 & 6,5 & 1,08 & & & & 8,5 & 6,5 & 1,31 \\
\hline 55 & & & 7 & 7 & 1 & & & & 8 & 8 & 1 \\
\hline 56 & & & 9 & 7,5 & 1,2 & & & & 8 & 7 & 1,14 \\
\hline 57 & & & 7,5 & 6,5 & 1,15 & & & & 7 & 6,5 & 1,08 \\
\hline 58 & & & 7,5 & 6,5 & 1,15 & & & & 7 & 7 & 1 \\
\hline 59 & & & 8 & 6,5 & 1,23 & & & & 7,5 & 6,5 & 1,15 \\
\hline 60 & & & 8 & 6,5 & 1,23 & & & & 7,5 & 7 & 1,07 \\
\hline 61 & & & 7,5 & 6,5 & 1,15 & & & & 7,5 & 6,5 & 1,15 \\
\hline 62 & & & 8,5 & 7 & 1,21 & & & & 8,5 & 7 & 1,21 \\
\hline 63 & & & 8 & 8 & 1 & & & & 8,5 & 7,5 & 1,13 \\
\hline 64 & & & 8 & 7,5 & 1,07 & & & & 8 & 6,5 & 1,23 \\
\hline 65 & & & 7,5 & 7,5 & 1 & & & & 9 & 7 & 1,29 \\
\hline 66 & & & 7,5 & 7,5 & 1 & & & & 9 & 7,5 & 1,2 \\
\hline 67 & & & 7,5 & 7 & 1,07 & & & & 7,5 & 7 & 1,07 \\
\hline 68 & & & 7 & 5,5 & 1,27 & & & & 7,5 & 5,5 & 1,36 \\
\hline 69 & & & 8,5 & 7 & 1,21 & & & & 7,5 & 6 & 1,25 \\
\hline 70 & & & 7,5 & 7 & 1,07 & & & & 7,5 & 6,5 & 1,15 \\
\hline 71 & & & 8 & 7,5 & 1,07 & & & & 8,5 & 6,5 & 1,31 \\
\hline 72 & & & 8 & 7 & 1,14 & & & & 8,5 & 6,5 & 1,31 \\
\hline 73 & & & 8 & 6,5 & 1,23 & & & & 9,5 & 7 & 1,36 \\
\hline 74 & & & 7,5 & 7 & 1,07 & & & & 8,5 & 6,5 & 1,31 \\
\hline 75 & & & 7,5 & 7 & 1,07 & & & & 8,5 & 7 & 1,21 \\
\hline 76 & & & 8 & 7 & 1,14 & & & & 9,5 & 6,5 & 1,46 \\
\hline 77 & & & 7,5 & 7 & 1,07 & & & & 9 & 7 & 1,29 \\
\hline 78 & & & 8 & 6,5 & 1,23 & & & & 7,5 & 6,5 & 1,15 \\
\hline 79 & & & 8,5 & 6,5 & 1,31 & & & & 9 & 7 & 1,29 \\
\hline 80 & & & 8 & 6 & 1,33 & & & & 7,5 & 7 & 1,07 \\
\hline 81 & & & 8 & 5,5 & 1,45 & & & & 9 & 7 & 1,29 \\
\hline 82 & & & 8 & 5,5 & 1,45 & & & & 8 & 6,5 & 1,23 \\
\hline 83 & & & 7 & 6,5 & 1,08 & & & & 8,5 & 6,5 & 1,31 \\
\hline 84 & & & 7 & 6,5 & 1,08 & & & & 7,5 & 6,5 & 1,15 \\
\hline 85 & & & 8,5 & 7 & 1,21 & & & & 7,5 & 7 & 1,07 \\
\hline 86 & & & 7,5 & 5,5 & 1,36 & & & & 9 & 7 & 1,29 \\
\hline 87 & & & 9 & 6,5 & 1,38 & & & & 8 & 8 & 1 \\
\hline 88 & & & 7,5 & 6,5 & 1,15 & & & & 8,5 & 7 & 1,21 \\
\hline 89 & & & 7 & 6 & 1,17 & & & & 8,5 & 7 & 1,21 \\
\hline 90 & & & 8 & 8 & 1 & & & & 7,5 & 7,5 & 1 \\
\hline 91 & & & 9 & 6,5 & 1,38 & & & & 7,5 & 6 & 1,25 \\
\hline 92 & & & 8 & 7,5 & 1,07 & & & & 8,5 & 6,5 & 1,31 \\
\hline
\end{tabular}


Table 3. (Continued).

\begin{tabular}{|c|c|c|c|c|c|c|c|c|c|c|c|c|}
\hline \multirow[b]{3}{*}{ No. } & \multicolumn{6}{|c|}{$\begin{array}{l}\text { Moesziomyces bullatus ex Echinochloa crus-galli, } \\
\text { GLM-F105814 }\end{array}$} & \multicolumn{6}{|c|}{$\begin{array}{l}\text { Moesziomyces bullatus ex Echinochloa crus-galli, } \\
\text { GLM-F105812 }\end{array}$} \\
\hline & \multicolumn{3}{|c|}{ sporeballs } & \multicolumn{3}{|c|}{ spores } & \multicolumn{3}{|c|}{ sporeballs } & \multicolumn{3}{|c|}{ spores } \\
\hline & length & breadth & $\mathrm{l} / \mathrm{b}$ & length & breadth & $\mathrm{l} / \mathrm{b}$ & length & breadth & $1 / b$ & length & breadth & $\mathrm{l} / \mathrm{b}$ \\
\hline 93 & & & & 8 & 7,5 & 1,07 & & & & 9 & 6,5 & 1,38 \\
\hline 94 & & & & 7,5 & 7,5 & 1 & & & & 7,5 & 6 & 1,25 \\
\hline 95 & & & & 7,5 & 6,5 & 1,15 & & & & 6,5 & 6 & 1,08 \\
\hline 96 & & & & 8 & 7,5 & 1,07 & & & & 7,5 & 7 & 1,07 \\
\hline 97 & & & & 7,5 & 7 & 1,07 & & & & 8,5 & 7,5 & 1,13 \\
\hline 98 & & & & 8,5 & 7 & 1,21 & & & & 7,5 & 6 & 1,25 \\
\hline 99 & & & & 10 & 7 & 1,43 & & & & 7,5 & 6,5 & 1,15 \\
\hline 100 & & & & 7,5 & 7,5 & 1 & & & & 8 & 7 & 1,14 \\
\hline
\end{tabular}

of Pseudozyma aphidis and the majority of $M$. bullatus isolates with the clade containing the type of $P$. rugulosa. Collections from $E$. crus-galli with smut symptoms were present in two different clades. The morphological investigation of a sexual morph from each clade (GLM-F105812 and GLM-F105814) revealed no morphological differences. Moesziomyces bullatus clustering within the majority of $M$. aphidis had the following spore characteristics: sporeballs variable in shape and size, 52.5-156.5 x 41.5-113 $\mu \mathrm{m}$, spores ovoid, globose, often irregular, pale yellow-brown, (6.5-)7-7.8-8.5(-10) $\times(5.5-) 6-6.8-7.5(-8) \mu \mathrm{m}$, a length/breadth ratio of $1.01-$ 1.15-1.39 ( $n=100)$ (Fig. 2). In comparison, the collection of $M$. bullatus clustering together with the sequence of the type species of $M$. rugulosus showed the following spore characteristics: sporeballs variable in shape and size, 58.5$144.5 \times 44-105 \mu \mathrm{m}$, spores ovoid, globose, often irregular, pale yellow-brown, (6-)7-7.9-8.5(-9.5) × (5.5-)6-6.7-7.5(8) $\mu \mathrm{m}$, a length/breadth ratio of 1.01-1.19-1.38 $(n=100)$ (Fig. 2). The sister group to $M$. bullatus was formed by $M$. antarcticus. The four lineages of $M$. bullatus formed an isolated clade with high to maximum support in all analyses together with samples classified as $M$. antarcticus.

Apart from the groups mentioned above, four additional distinct groups were revealed. Two of these corresponded to lineages formed by sexual smuts of the genus Moesziomyces isolated from plants with smut disease symptoms. One of these corresponded to $M$. bullatus s. lat. on Paspalum distichum, and the other to Eriomoeszia eriocauli. The remaining two lineages formed a monophyletic clade with high support in Minimum Evolution Analysis. One lineage included sequences of yeasts classified as M. parantarcticus, the other a sexual morph of a plant pathogenic fungus of the genus Moesziomyces from Pennisetum glaucum, as well asexual morphs isolated from symptom-free barley and a preterm infant.

\section{DISCUSSION}

Moesziomyces is a morphologically well-defined genus in the smut fungi, mainly characterised by ruptured sterile cells in the sori around the spores. The genus was believed to be monotypic by Vánky (2012), but phylogenetic investigations of the past decade have shown that several species previously assigned to the asexually typified yeast genus Pseudozyma, were closely related to Moesziomyces bullatus (Begerow et al. 2000, 2006, Wang et al. 2006, 2015). In the latest edition of the International Code of Nomenclature for algae, fungi and plants (ICN) it is ruled that the dual naming for asexual and sexual morphs of fungi has been discontinued (McNeill et al. 2012). Consequently, Wang et al. (2015) attempted to resolve the names of species placed in the genus Pseudozyma as far as possible, and combined those related to Moesziomyces bullatus s.lat. into Moesziomyces.

The yeast asexual morphs were, for example, found to live epiphytically on different hosts (Boekhout 1995), but also to occur on a variety of other substrates. Due to their asexual reproduction with pullulating and division, it is possible for them to colonize suitable habitats in a short period of time. Of these yeasts, Pseudozyma aphidis is often considered as a biocontrol agent for plant pathogenic fungi (Avis et al. 2001, Buxdorf et al. 2013). Thus it is noteworthy that one isolate of this species co-occurred in Albugo laibachii lesions on Arabidopsis thaliana, indicating only no or only limited antagonism against this specialised white blister rust species (Thines et al. 2009). It is commonly believed that most Pseudozyma species have lost pathogenicity, which is seemingly supported by recent genomic analyses (Lefebvre et al. 2013). However, it should be noted that if a different start codon is taken for translation than the one predicted, all Pseudozyma yeasts included by Lefebvre et al. (2013) have a functional copy of PEP1, a conserved effector among smut fungi of the Ustilaginales (Sharma et al. 2014, Hemetsberger et al. 2015), suggesting the possibility of a misannotation of the start codon. In-depth bioinformatic analyses and functional testing will be needed to clarify this situation.

Deducing the conspecificity of Moesziomyces bullatus with Pseudozyma aphidis and $P$. rugulosa was not possible for Wang et al. (2015), as they did not include sequences from the type host of M. bullatus, Echinochloa crus-galli, but only from $M$. verrucosus on Paspalum distichum, which they erroneously assumed to be conspecific with $M$. bullatus. However, the smut sexual morphs from the type host, E. crusgalli from Germany, are placed in two of the four subclusters 


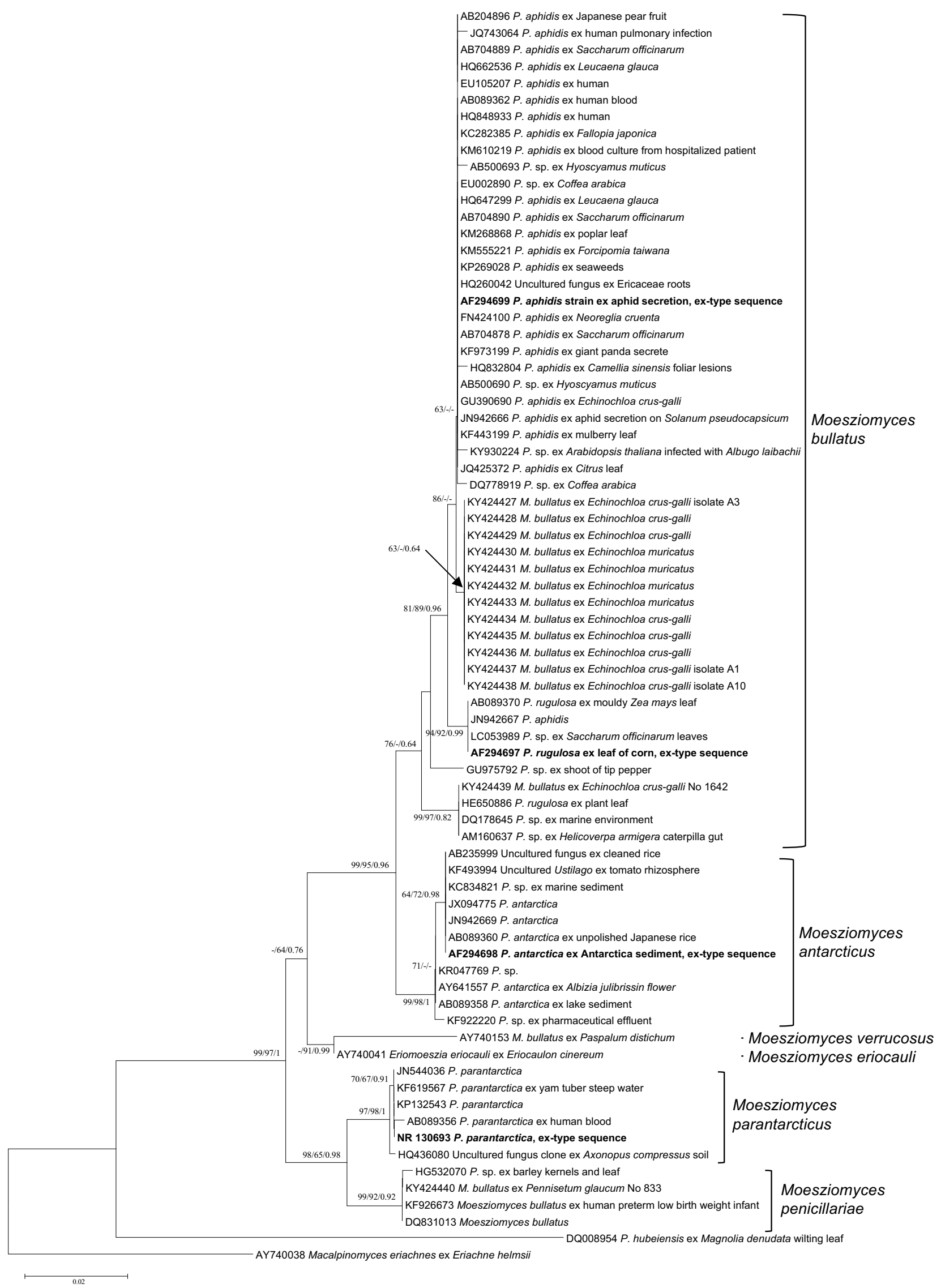

Fig. 1. Phylogenetic tree based on Minimum Evolution analyses of nrlTS sequences of Moesziomyces spp., rooted with Macalpinomyces eriachnes. Numbers on branches denote bootstrap support in Minimum Evolution, Maximum Likelihood and a posteriori probabilities from Bayesian Analyses. Values below $55 \%$ are not shown. The bar indicates expected substitutions per site. GenBank numbers precede taxon names, and are followed by the name of the host or isolation source of the fungus. 

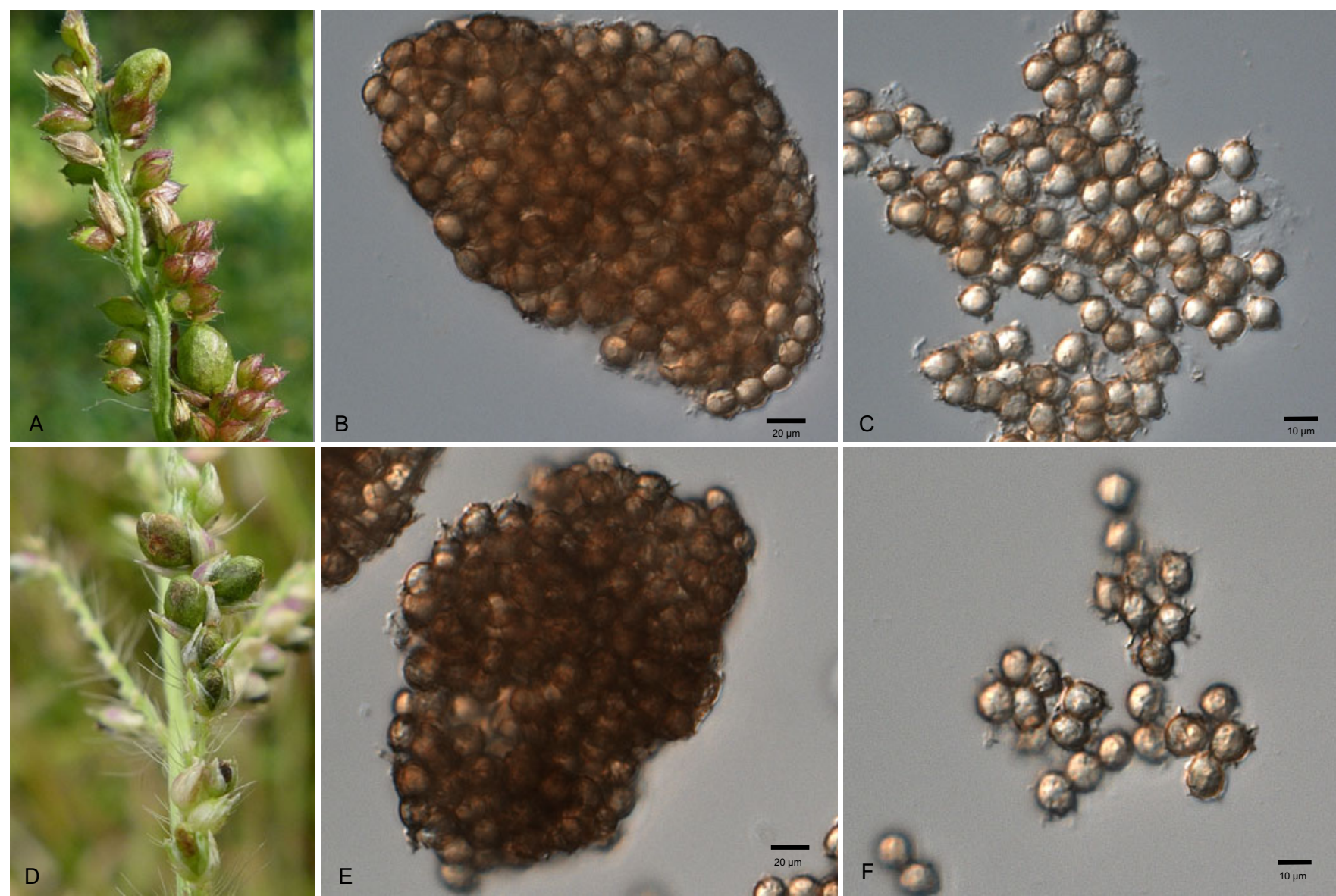

Fig. 2. Sori and spores of Moesziomyces bullatus on Echinochloa crus-galli. A, D. Sori. B, E. Teliospore balls. C, F. Teliospores. A-C (GLM-F105812), D-F (GLM-F105814).

of a larger cluster, which is interpreted here as representing $M$. bullatus. It is noteworthy that three of the four subclusters of $M$. bullatus contain environmental samples from various sources. In conjunction with the ease of cultivation observed for M. bullatus from E. crus-galli, it is concluded that unlike the vast majority of genera of Ustilaginales, the asexual yeast morph plays a major role as a proliferating life-cycle stage in Moesziomyces and that the plant-parasitic dikaryophase is probably mainly important for maintaining the possibility of sexual recombination. As the two subclades previously referred to as $M$. aphidis and $M$. rugulosus are interspersed with the morphologically identically lineages of $M$. bullatus from $E$. crus-galli, they are probably better included in $M$. bullatus until more sequence data become available. It seems probable that, with the inclusion of additional smut samples from Echinochloa, additional smut-causing members of the subclades will be discovered. Sampling in Africa seems to be promising in this respect, as the species diversity of Echinochloa is highest on this continent. Also, the notion that yeasts of the subclade containing the ex-type culture of Pseudozyma aphidis can withstand high temperatures, such as the human body temperature, is suggestive of a subtropical to tropical origin of this lineage.

Further, our investigations show that the older name $M$. eriocauli for Eriomoeszia eriocauli, should be taken up again, as this species was found embedded within Moesziomyces.

With the synonymy of the generic name Eriomoeszia and the reappraisal of the hardly used Moesziomyces names of the smut fungi of Paspalum and Pennisetum, Moesziomyces now includes six species. It is, however, likely that additional species will have to be added because smut samples from some Poaceae genera listed as host plants for the $M$. bullatus complex in Vánky (2012) could not be included in the current study, such as smuts from Leersia, Panicum, Polytrias, and Uranthoecium. Given the apparently high host specificity of Moesziomyces species, it seems likely that these pathogens represent species independent from $M$. bullatus.

\section{TAXONOMY}

Based on the phylogenetic data presented here, the following nomenclature and taxonomic changes are made.

Moesziomyces antarcticus (Goto et al.) Q.M. Wang et al., Stud. Mycol. 81: 81 (2015).

Basionym: Sporobolomyces antarcticus Goto et al., Mycologia 61: 759 (1969).

Synonyms: Candida antarctica (Goto et al.) Kurtzman et al., Yeasts: 86 (1983).

Vanrija antarctica (Goto et al.) R.T. Moore, Bibltheca Mycol. 108: 167 (1987).

Pseudozyma antarctica (Goto et al.) Boekhout, J. Gen. Appl. Microbiol. 41: 364 (1995). 
Moesziomyces bullatus (J. Schröt.) Vánky, Bot. Notiser 130: 133 (1977).

Basionym: Sorosporium bullatum J. Schröt., Abh. Schles. Ges. Vaterl. Cult., Abth. Naturwiss. 72: 6 (1869).

Synonyms: Tolyposporium bullatum J. Schröt, in Cohn., Krypt. Fl. Schles. 3(1): 276 (1887).

Sterigmatomyces aphidis Henninger \& Windisch, Arch. Microbiol. 105: 50 (1975).

Tolypoderma bullata (J. Schröt.) Thirum. \& M.J. O`Brien, Friesia 11: 190 (1978) ["1977“].

Sporothrix rugulosa Traquair et al., Canad. J. Bot. 66: 929 (1988).

Stephanoascus rugulosus Traquair et al., Canad. J. Bot. 66: 929 (1988).

Pseudozyma aphidis (Henninger \& Windisch) Boekhout, $J$. Gen. Appl. Microbiol. 41: 364 (1995).

Pseudozyma rugulosa (Traquair, et al.) Boekhout \& Traquair, J. Gen. Appl. Microbiol. 41: 364 (1995).

Moesziomyces aphidis (Henninger \& Windisch) Q.M. Wang et al., Stud. Mycol. 81: 81 (2015).

Moesziomyces rugulosus (Traquair, et al.) Q.M. Wang et al., Stud. Mycol. 81: 81 (2015).

Moesziomyces eriocauli (G.P. Clinton) Vánky, Nordic J. Bot. 6: 71 (1986).

Basionym: Tolyposporium eriocauli G.P. Clinton, Rhodora 2: 82 (1901).

Synonyms: Dermatosorus eriocauli (G.P. Clinton) M.D. Whitehead \& Thirum., Mycologia 64: 128 (1972).

Tolypoderma eriocauli (G.P. Clinton) Thirum., Friesia 11: 191 (1978).

Eriomoeszia eriocauli (G.P. Clinton) Vánky, Mycol. Balcanica 2: 106 (2005).

Moesziomyces parantarcticus (Sugita et al.) Q.M. Wang et al., Stud. Mycol. 81: 81 (2015).

Basionym: Pseudozyma parantarctica Sugita et al., Microbiol. Immun. 47: 186 (2003).

Moesziomyces verrucosus (J. Schröt.) J. Kruse \& Thines, comb. nov.

MycoBank MB819410

Basionym: Ustilago verrucosa J. Schröt., Hedwigia 35: 214 (1896).

Synonyms: Tolyposporium evernium Syd., Ann. Mycol. 37: 443 (1939).

Tolyposporium paspali Langdon, Univ. Queensland Dept. Biol. Pap 2(9): 4 (1948).

Moesziomyces evernius (Syd.) Vánky, Bot. Notiser 130: 135 (1977).

Tolyposporidium evernium (Syd.) Thirum. \& Neerg., Friesia 11: 180 (1978) [“1977”].

Moesziomyces penicillariae (Bref.) Vánky, Bot. Notiser 130: 135 (1977).

Basionym: Tolyposporium penicillariae Bref., Unters. Gesammtgeb. Mykol. 12: 154 (1895).

Synonym: Tolyposporidium penicillariae (Bref.) Thirum. \& Neerg., Friesia 11: 181 (1978) [“1977”].

\section{ACKNOWLEDGEMENTS}

This study was supported by LOEWE in the framework of the excellence cluster for Integrative Fungal Research (IPF). We thank B. Mishra and colleagues for the possibility to use the TrEase Webserver at www.thines-lab.senckenberg.de/trease prior to publication. $U$. Damm is greatfully acknowledged for her help with depositing specimens in GLM.

\section{REFERENCES}

Arendrup MC, Boekhout T, Akova M, Meis JF, Cornely OA, et al. (2014) ESCMID and ECMM joint clinical guidelines for the diagnosis and management of rare invasive yeast infections. Clinical Microbiology and Infection Supplement 3: 76-98.

Avis TJ, Caron SJ, Boekhout T, Hamelin RC, Bélanger RR (2001) Molecular and physiological analysis of the powdery mildew antagonist Pseudozyma flocculosa and related fungi. Phytopathology 91: 249-254.

Babajide JM, Maina S, Kiawa B, Skilton R (2015) Identification of fungal isolates from steeped yam (Gbodo): Predominance of Meyerozyma guilliermondii. Food Science and Biotechnology: 24: 1041-1047.

Bandoni RJ (1985) On an undescribed, pleomorphic hyphomycete from litter. Botanical Journal of the Linnean Society 91(1-2): 37-43.

Begerow D, Stoll M, Bauer R (2006) A phylogenetic hypothesis of Ustilaginomycotina based on multiple gene analyses and morphological data. Mycologia 98: 906-916.

Begerow D, Bauer R, Boekhout T (2000) Phylogenetic placements of ustilaginomycetous anamorphs as deduced from nuclear LSU rDNA sequences. Mycological Research 104: 53-60.

Boekhout T (1995) Pseudozyma Bandoni emend. Boekhout, a genus for yeast-like anamorphs of Ustilaginales. Journal of General and Applied Microbiology Tokyo 41: 359-366.

Boekhout T (2011) Pseudozyma Bandoni emend. Boekhout (1985) and a comparison with the yeast state of Ustilago maydis (De Candolle) Corda (1842). In: The Yeasts, a Taxonomic Study (Kurtzman CP, Fell JW, Boekhout T, eds): 1857-1868. Amsterdam: Elsevier.

Buxdorf K, Rahat I, Gafni A, Levy M (2013) The epiphytic fungus Pseudozyma aphidis induces jasmonic acid-and salicylic acid/ nonexpressor of PR1-independent local and systemic resistance. Plant Physiology 161: 2014-2022.

Chang MH, Kim HJ, Jahng KY, Hong SC (2008) The isolation and characterization of Pseudozyma sp. JCC 207, a novel producer of squalene. Applied Microbiology and Biotechnology 78: 963.

Escudero, M (2015) Phylogenetic congruence of parasitic smut fungi (Anthracoidea, Anthracoideaceae) and their host plants (Carex, Cyperaceae): cospeciation or host-shift speciation? American Journal of Botany 102: 1108-1114.

Gafni A, Calderon CE, Harris R, Buxdorf K, Dafa-Berger A, et al. (2015) Biological control of the cucurbit powdery mildew pathogen Podosphaera xanthii by means of the epiphytic fungus Pseudozyma aphidis and parasitism as a mode of action. Frontiers in Plant Science 6: 1-11.

Han PJ, Qiu JZ, Wang QM, Bai FY (2012) Udeniomyces kanasensis sp. nov., a ballistoconidium-forming yeast species in the Cystofilobasidiales. Antonie van Leeuwenhoek: 102: 45-51. 
Hawksworth DL, Crous PW, Redhead SA, Reynold DR, Samson $\mathrm{RA}$, et al. (2011) The Amsterdam Declaration on Fungal Nomenclature. IMA Fungus 2: 105-112.

Hemetsberger C, Mueller AN, Matei A, Herrberger C, Hensel G, et al. (2015) The fungal core effector Pep1 is conserved across smuts of dicots and monocots. New Phytologist 206: 1116-1126.

Ikeda S, Fuji SI, Sato T, Furuya H, Naito H, et al. (2007) Microbial diversity in milled rice as revealed by ribosomal intergenic spacer analysis. Microbes and Environments 22: 165-174.

Irinyi L, Serena C, Garcia-Hermoso D, Arabatzis M, Desnos-Ollivier $M$, et al. (2015) International Society of Human and Animal Mycology (ISHAM)-ITS reference DNA barcoding databasethe quality controlled standard tool for routine identification of human and animal pathogenic fungi. Medical Mycology 53: 313-317.

Kämper J, Kahmann R, Bölker M, Ma LJ, Brefort T, et al. (2006) Insights from the genome of the biotrophic fungal plant pathogen Ustilago maydis. Nature 444: 97-101.

Katoh K, Standley DM (2013) MAFFT multiple sequence alignment software version 7 : improvements in performance and usability. Molecular Biology and Evolution 30: 772-780.

Korhola M, Hakonen R, Juuti K, Edelmann M, Kariluoto S, et al. (2014) Production of folate in oat bran fermentation by yeasts isolated from barley and diverse foods. Journal of Applied Microbiology 117: 679-689.

Kruse J, Choi Y J, Thines M (2017) New smut-specific primers for the ITS barcoding of Ustilaginomycotina. Mycological Progress 16: 213-221.

Lefebvre F, Joly DL, Labbé C, Teichmann B, Linning R, et al. (2013) The transition from a phytopathogenic smut ancestor to an anamorphic biocontrol agent deciphered by comparative wholegenome analysis. Plant Cell 25: 1946-1959.

Li YM, Shivas RG, Cai L (2017) Cryptic diversity in Tranzscheliella spp. (Ustilaginales) is driven by host switches. Scientific Reports: 7: 43549.

Lin SS, Pranikoff T, Smith SF, Brandt ME, Gilbert K, et al. (2008) Central venous catheter infection associated with Pseudozyma aphidis in a child with short gut syndrome. Journal of Medical Microbiology 57: 516-518.

Matheny PB, Gossmann JA, Zalar P, Kumar TA, Hibbett DS (2006) Resolving the phylogenetic position of the Wallemiomycetes: an enigmatic major lineage of Basidiomycota. Botany: 84: 17941805.

McNeill J, Barrie FF, Buck WR, Demoulin V, Greuter W, et al. (eds) (2012) International Code of Nomenclature for algae, fungi and plants (Melbourne Code). [Regnum vegetabile no. 154.] Königstein: Koeltz Scientific Books.

McNeil JC, Palazzi DL (2012) Ustilago as a cause of fungal peritonitis: case report and review of the literature. Journal of the Pediatric Infectious Diseases Society 1: 337-339.

McTaggart AR, Shivas RG, Geering ADW, Callaghan B, Vánky $\mathrm{K}$, et al. (2012) Soral synapomorphies are significant for the systematics of Sporisorium. Persoonia 29: 63-77.

Moore M, Russell WO, Sachs E (1946) Chronic leptomeningitis and ependymitis caused by Ustilago, probably U. zeae (corn smut). American Journal of Pathology 22: 761-777.

Morita T, Fukuoka T, Imura T, Hirose N, Kitamoto D (2012) Isolation and screening of glycolipid biosurfactant producers from sugarcane. Bioscience, Biotechnology, and Biochemistry 76: 1788-1791.
Okolo OM, Van Diepeningen AD, Toma B, Nnadi NE, Ayanbimpe MG, et al. (2015) First report of neonatal sepsis due to Moesziomyces bullatus in a preterm low-birth-weight infant. JMM Case Reports 2(2): doi: 10.1099/jmmcr.0.000011.

Parahym AM, da Silva CM, Domingos Ide F, Gonçalves SS, Rodrigues Mde M, et al. (2013) Pulmonary infection due to Pseudozyma aphidis in a patient with Burkitt lymphoma: first case report. Diagnostic Microbiology and Infectious Disease 75: 104-106.

Prakash A, Wankhede S, Singh PK, Agarwal K, Kathuria S, et al. (2014) First neonatal case of fungemia due to Pseudozyma aphidis and a global literature review. Mycoses 57: 64-68.

Sampaio JP (2004) Diversity, phylogeny and classification of basidiomycetous yeasts. In: Frontiers in Basidiomycote Mycology (Agerer R, Blanz P, Piepenbring M, eds): 49-80. Eching: IHW-Verlag.

Sharma R, Mishra B, Runge F, Thines M (2014) Gene loss rather than gene gain is associated with a host jump from monocots to dicots in the smut fungus Melanopsichium pennsylvanicum. Genome Biology and Evolution 6: 2034-2049.

Shetty HS, Safeeulla KM (1979) Stimulation of teliospore germination in smut fungi. Proceedings of the Indian Academy of Science 88: 479-486.

Shivas RG, Lutz M, McTaggart AR, Vánky K (2013) Emended description of Anomalomyces (Ustilaginales) including Anomalomyces yakirrae sp. nov. on Yakirra pauciflora (Poaceae) from Australia. Mycobiota 1: 17-24.

Stoll M, Begerow D, Oberwinkler F (2005) Molecular phylogeny of Ustilago, Sporisorium, and related taxa based on combined analyses of rDNA sequences. Mycological Research 109: 342356.

Stoll M, Piepenbring M, Begerow D, Oberwinkler F (2003) Molecular phylogeny of Ustilago and Sporisorium species (Basidiomycota, Ustilaginales) based on internal transcribed spacer (ITS) sequences. Canadian Journal of Botany 81: 976-984.

Sugita T, Takashima M, Poonwan N, Mekha N, Malaithao K, et al. (2003) The first isolation of ustilaginomycetous anamorphic yeasts, Pseudozyma species, from patients' blood and a description of two new species: $P$. parantarctica and $P$. thailandica. Microbiology and Immunology 47: 183-190.

Tamura K, Stecher G, Peterson D, Filipski A, Kumar S (2013) MEGA6: Molecular Evolutionary Genetics Analysis Version 6.0. Molecular Biology and Evolution 30: 2725-2729.

Teo L H, Tay Y K (2006) Ustilago species infection in humans. British Journal of Dermatology 155: 1096-1097.

Valverde ME, Paredes-Lopez O, Pataky JK, Guevara-Lara F (1995) Huitlacoche (Ustilago maydis) as a food source-biology, composition and production. Critical Reviews in Food Science and Nutrition 35: 191-229.

Vánky K (1977) Moesziomyces, a new genus of Ustilaginales. Botaniska Notiser 130: 131-135.

Vánky K (1986) The genus Moesziomyces (Ustilaginales). Nordic Journal of Botany 6: 67-73.

Vánky K (2005) The smut fungi (Ustilaginomycetes) of Eriocaulaceae. I. Eriomoeszia gen. nov. Mycologia Balcanica 2: 105-111.

Vánky K (2012) Smut Fungi of the World. St Paul, MN: American Phytopathological Society Press.

Wang Q-M, Jia J-H, Bai F-Y (2006) Pseudozyma hubeiensis sp. nov. and Pseudozyma shanxiensis sp. nov., novel ustilaginomycetous anamorphic yeast species from plant leaves. International 
Journal of Systematic and Evolutionary Microbiology 56: 289293.

Vega FE, Posada F, Aime MC, Peterson SW, Rehner SA (2008) Fungal endophytes in green coffee seeds. Mycosystema 27: 75-84.

Walker JF, Aldrich-Wolfe L, Riffel A, Barbare H, Simpson NB, et al. (2011) Diverse Helotiales associated with the roots of three species of Arctic Ericaceae provide no evidence for host specificity. New Phytologist 191: 515-527.

Wang Q.-M, Begerow D, Groenewald M, Liu X-Z, Theelen B, et al. (2015) Multigene phylogeny and taxonomic revision of yeasts and related fungi in the Ustilaginomycotina. Studies in Mycology 81: 55-83.

Wei YH, Fwu-Ling L, Wen-Haw H, Shyue-Ru CHEN, Chien-Cho CHEN, et al. (2005) Pseudozyma antarctica in Taiwan: a description based on morphological, physiological and molecular characteristics. Botanical Bulletin of Academia Sinica 46: 223229.
Wei YH, Liou GY, Lee FL (2011) Pseudozyma aphidis, a new record of ustilaginomycetous anamorphic fungi in Taiwan. Fungal Science 26: 1-5.

White TJ, Bruns T, Lee S, Taylor JW (1990) Amplification and direct sequencing of fungal ribosomal RNA genes for phylogenetics. In: PCR Protocols: a guide to methods and applications (Innis MA Gelfand DH, Sninsky JJ, White TJ, eds): 315-322. San Diego: Academic Press.

Yasuda F, Yamagishi D, Izawa H, Kodama M, Otani H (2007) Fruit stain of Japanese pear caused by basidiomycetous, yeastlike fungi Meira geulakonigii and Pseudozyma aphidis. Japanese Journal of Phytopathology 73: 166-171. 